of environmental justice. This includes engaging stakeholders at all stages of the production process and at all locations along the commodity chain, to ensure that unjust power structures are not reproduced due to the technology's 'green halo'.

This text is an example of high-calibre interdisciplinarity, both accessible and meticulously researched. Students in environmental studies, energy policy and environmental sociology will find it an in-depth primer from which they can engage in further investigation through the extensive resources provided. Those completely new to solar power technologies and their social, political and environmental context may need to reread paragraphs at times due to their richness, but ultimately will emerge able to engage with the professional and academic energy community at a high level of sophistication.

While beyond the scope of this text, it is worth recognizing that solar, despite its rapid growth, remains an extremely small part of the energy grid (1.6\% in 2018, according to the US Energy Information Administration). The thorough assessment of the broader impacts of solar, per Mulvaney's text, are well warranted. However, when evaluating sources of US electricity generation, the sector remains dominated by natural gas, coal and nuclear, the impacts of which do not yet appear to have been considered in such a rigorous and interdisciplinary fashion. Mulvaney's approach to solar development provides a template for how this type of research could - and undoubtedly should be applied to other types of electricity generation, especially those that reduce carbon emissions and for whom growth is anticipated. That said, to provide a primer that is aspirational, critical and meticulously researched is no easy task. Solar Power achieves just that.

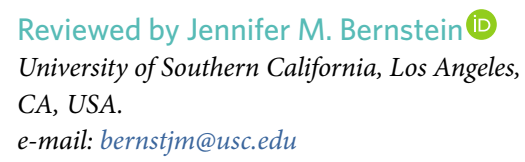

Published online: 26 July 2019

https://doi.org/10.1038/s41558-019-0532-7

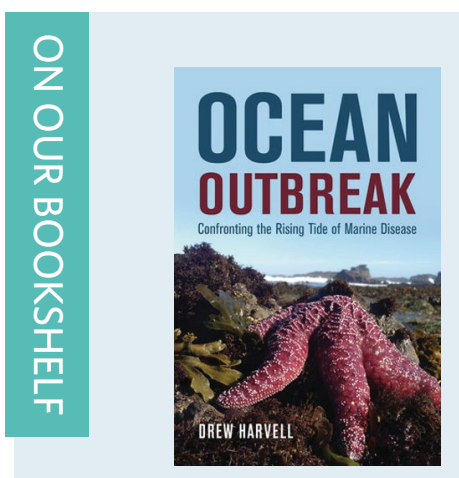

\title{
Ocean Outbreak: Confronting the Rising Tide of Marine Disease
}

By Drew Harvell

UNIVERSITY OF CALIFORNIA PRESS: 2019. 232PP. US\$26.95

Harvell offers a firsthand account of her investigations into marine diseases attacking corals, abalone, salmon and starfish, delivering the message that major marine epidemics can be remedied with policy changes and innovative solutions.

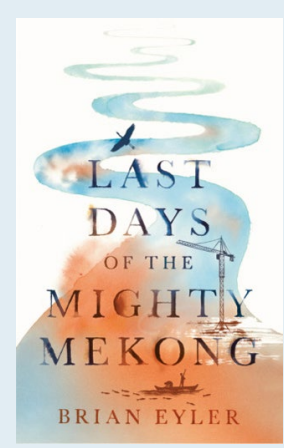

\section{Last Days of the Mighty Mekong \\ By Brian Eyler}

ZED BOOKS: 2019. 163PP. US\$25.00

Rapid industrialization and urban development are transforming the Mekong River that runs from China to Southern Vietnam. Eyler takes us on a journey through the natural wonders, people and policies shaping this unique ecosystem.

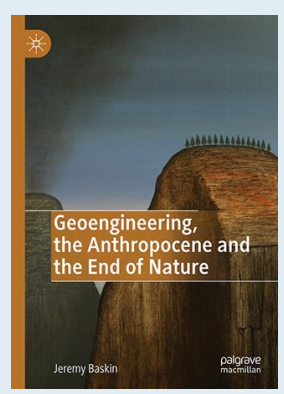

\section{Geoengineering, the Anthropocene and the End of Nature}

By Jeremy Baskin

PALGRAVE MACMILLAN: 2019. 217PP. £59.99

Baskin takes a critical look at the past, present and future of solar geoengineering as an idea, arguing that it is not just a technology, but a sociotechnical project wrapped up in varied and competing sets of values, institutions and symbols.

Published online: 26 July 2019

https://doi.org/10.1038/s41558-019-0547-0 